\title{
NÃO OBTENÇÃO DO CATETERISMO INTRAVENOSO PERIFÉRICO NA PRIMEIRA TENTATIVA NUMA UNIDADE DE ONCOLOGIA PEDIÁTRICA
}

\section{Isana Louzada Brito Santos; Luciano Marques dos Santos²; Cleonara Souza Gomes e Silva $^{3}$}

1. Voluntária PEVIC/UEFS, Graduando em Enfermagem, Universidade Estadual de

Feira de Santana, e-mail: isanalouzada@hotmail.com

2. Orientador, Departamento de Saúde, Universidade Estadual de Feira de Santana, email: lucmarxenfo@yahoo.com.br

3. Participante do Projeto "Segurança do paciente pediátrico e sua família: estudo de tecnologias e eventos adversos relacionados à terapia intravascular periférica", Universidade Estadual de Feira de Santana, e-mail: cleosilvauefs@ gmail.com

PALAVRAS-CHAVE: Enfermagem pediátrica; Cateterismo periférico; Enfermagem oncológica.

\section{INTRODUÇÃO}

A internação hospitalar é uma das experiências mais difíceis na vida de uma criança. Gera ansiedade, pois implica no afastamento da família, impede o convívio social, provoca mudança de rotina e restrições para brincar no hospital. Há diversas situações que podem levar a criança a ser hospitalizada, dentre elas, destaca-se o diagnóstico de câncer.

Dentre as modalidades terapêuticas existentes para o tratamento do câncer a quimioterapia é a mais difundida. A via mais utilizada para sua administração é a intravenosa (ANDRADE; SILVA, 2007). Assim, a criança hospitalizada para o diagnóstico e tratamento de câncer é exposta a procedimentos invasivos, inevitáveis e potencialmente dolorosos, com destaque para o cateterismo intravenoso periférico (CIP).

O CIP consiste na inserção de um dispositivo no interior de uma veia com o objetivo de promover infusão de drogas, fluidos, dieta parenteral ou coleta de sangue. Esse procedimento é de responsabilidade da equipe de enfermagem, realizado predominantemente por profissionais de nível técnico, e cabe a eles utilizarem técnicas adequadas no preparo da criança e na inserção do cateter (GOMES et al., 2010).

Crianças em uso de quimioterápicos, medicações de natureza irritante ou vesicante tendem a desenvolver uma fragilidade no endotélio vascular, fato que pode dificultar o sucesso do CIP na primeira tentativa (HASS, 2004). Desta forma, a identificação de crianças com dificuldade de obtenção do acesso intravenoso periéfico AIP pode auxiliar os profissionais a utilizarem outros recursos para reduzir o número de tentativas de punção.

A tentativa de CIP é definida por Yen, Riegert e Gorelick (2008), como a primeira vez que a agulha toca a pele e quando a agulha é removida da pele. O sucesso do CIP é identificado quando há retorno do fluxo sanguíneo e quando uma solução salina pode ser introduzida sem comprometer a veia, assim, o acesso está pronto para receber fluido e / ou medicação.

Os fatores relacionados a não obtenção do CIP na primeira tentativa não estão bem estabelecidos na literatura nacional e internacional. Desta forma, este estudo teve como objetivo identificar os fatores predisponentes para a não obtenção do CIP em crianças que são tratadas na clínica oncológica de um hospital no interior da Bahia.

\section{MATERIAIS E MÉTODOS}

Estudo transversal, realizado em um hospital pediátrico de Feira de Santana-BA, com uma amostra por conveniência de 130 CIP em crianças que obtiveram ou não o AIP na primeira tentativa. Os dados foram coletados no banco de dados do Projeto intitulado 
"Segurança do paciente pediátrico e sua família: estudo de tecnologias e eventos adversos relacionados à terapia intravascular periférica" (SANTOS, 2015) mediante a autorização do coordenador. Os dados foram processados no Statistical Package for the Social Sciences (SPSS) versão 22.0, e as variáveis descritas em frequências absolutas e relativas. Os testes de hipóteses estatísticos utilizados foram o Qui-quadrado de Pearson e o Teste Exato de Fisher, a análise multivariada foi do tipo regressão logística. Para as duas análises foram adotados como nível de significância os valores iguais ou inferiores a 0,05.

\section{RESULTADOS E DISCUSSÃO}

A taxa de não obtenção do CIP na primeira tentativa foi de 38,5\%. Foi verificado que as seguintes variáveis apresentaram associação estatística com a não obtenção do CIP na primeira tentativa: agitação da criança $(\mathrm{p}=0,005)$, histórico de dificuldade para a realização do CIP $(\mathrm{p}=0,035)$, tempo de hospitalização $(\mathrm{p}=0,005)$, uso de TIV periférica prolongada $(\mathrm{p}=0,005)$ e antecedente de extravasamento $(\mathrm{p}=0,008)$.

A regressão logística mostrou que foi confirmada associação estatística apenas para as variáveis: agitação da criança durante o $\operatorname{CIP}(p=0,005)$, tempo de hospitalização $(p=0,001)$, uso de TIV periférica prolongada $(\mathrm{p}=0,001)$ e antecedente de extravasamento $(\mathrm{p}=0,001)$.

Nesse estudo, $51,7 \%$ das crianças demonstraram agitação no momento do CIP, havendo associação estatisticamente significante nessa variável, as crianças que apresentaram agitação tiveram prevalência aproximadamente 2 vezes maior para a não obtenção do CIP na primeira tentativa.

Embora a experiência e competência do enfermeiro tenham sido correlacionadas com a obtenção do AIP na primeira tentativa, a cooperatividade da criança mostrou-se um melhor preditor de sucesso. Desta forma, quando a criança apresenta agitação durante o procedimento, o CIP se torna mais difícil de ser conseguido na primeira tentativa (LININGER, 2003; LARSEN et al., 2010).

A agitação da criança pode estar associada à falta de informação que ela recebe da equipe de enfermagem. Geralmente o CIP ocorre sem uma preparação prévia e isso pode potencializar o medo e as reações de estresse, dificultando a obtenção do AIP na primeira tentativa (BEZERRA et al., 2009). Na unidade que esta pesquisa foi realizada, não é utilizado nenhum método de preparação das crianças para o CIP. Portanto, é interessante que sejam incorporadas à prática diária dos profissionais, tecnologias de preparo prévio da criança para o CIP, já que são constatadas por evidências científicas com métodos eficazes para a redução da inquietação da criança durante o procedimento (GUARISE et al., 2010).

As crianças que passaram mais de 8 dias hospitalizadas possuíram 1,87 vezes mais prevalência de não obter o CIP na primeira tentativa, essa variável apresentou associação estatisticamente significante. Isso pode estar associado à TIV instituída para a criança durante esse período de hospitalização, pois geralmente quanto mais dias a criança tem de hospitalização, mais tempo de TIV ela terá.

Um estudo realizado por Bensghir et al. (2012) revelou em seus resultados que a hospitalização por um tempo maior que 5 dias $(\mathrm{p}=0,028)$ é um preditor de dificuldade para a realização do CIP. Entretanto, não foram encontrados estudos que relacionem o tempo de hospitalização com a não obtenção do CIP na primeira tentativa.

Houve associação estatisticamente significante para TIV prévia prolongada e essas crianças apresentaram prevalência de 1,87 maior de não obter o CIP na primeira tentativa. Vezzani (2013) afirma que a TIV prévia prolongada é um fator de risco para a dificuldade de obter o AIP.

O histórico de dificuldade para a realização do CIP foi estatisticamente significante nesta pesquisa, apresentando prevalência de 1,62 vezes maior para crianças que tiveram 
dificuldade de obtenção do AIP, corroborando estudos que trazem que essa variável é um fator de risco para a não obtenção do CIP na primeira tentativa (LOON et al., 2016).

Esse resultado torna-se preocupante, pois quando há dificuldade de obtenção do CIP, há uma tendência dos profissionais escolherem a veia mais fácil para realizar a cateterização. Entretanto, nem sempre a veia mais fácil é a mais indicada para iniciar a TIV, principalmente se tratando de quimioterápicos, que geralmente tem a natureza irritante ou vesicante e pode causar complicações, a exemplo de flebite, infiltração, extravasamento ou obstrução.

Ao contrário do que foi encontrado no estudo de Negri et al. (2012), nesta pesquisa, os antecedentes de complicação não apresentaram associação estatisticamente significante, quando analisados em conjunto. Entretanto, as crianças que tiveram antecedentes de extravasamento apresentaram prevalência 1,95 vezes maior de não obtenção do CIP na primeira tentativa.

Essas complicações podem ocorrer, inclusive, por fatores físicos, desencadeados pela técnica do CIP, características anatômicas da veia, pela doença de base do indivíduo e características do dispositivo intravenoso (PEREIRA; ZANETTI, 2000). Portanto, merecem atenção especial, pois além de ser um fator que pode contribuir para a não obtenção do CIP na primeira tentativa, pode causar repercussões na qualidade de vida da criança, principalmente quando se trata de extravasamento, que pode provocar lesões teciduais sérias, causando mais dor e desconforto à criança.

Houve significância epidemiológica para a visibilidade da veia, as crianças que não possuíam o trajeto venoso visível no momento do CIP apresentaram prevalência 1,55 vezes maior para a não obtenção do AIP na primeira tentativa, sendo compatível com estudos realizados em adultos e crianças (RIKER et al., 2011; LOON et al., 2016). A presença de uma veia visível $(p<0,001)$ e a presença de uma veia palpável $(p=0,015)$ foram preditores significativos de obtenção do CIP na primeira tentativa (CARR et al., 2016b).

As limitações metodológicas desse estudo consistem em não ter sido realizado o cálculo estatístico da amostra devido à ausência de estudos nacionais ou internacionais nessa população, ou na própria unidade estudada, portanto, a amostra foi escolhida por conveniência. A amostra da pesquisa foi baseada no número de CIP coletado e não no número de crianças participantes do estudo, podendo uma mesma criança ter sido abordada pela equipe de coleta mais de uma vez.

\section{CONCLUSÃO}

Foi constatado através do estudo que foram encontradas variáveis estatisticamente significantes para a não obtenção do CIP na primeira tentativa. Os resultados desse estudo possuem relevância social e científica, já que poderá preencher lacunas na literatura nacional e internacional, servindo como fonte de pesquisa e como evidência científica para a transformação da prática clínica dos profissionais envolvidos na implementação da TIV em crianças oncológicas. Poderá também estimular debates dentro das instituições de ensino a respeito de estratégias para promover segurança ao paciente pediátrico.

Essa pesquisa irá beneficiar as crianças e suas famílias que terão o sofrimento reduzido com o aumento das taxas de sucesso do CIP na primeira tentativa, tornando o tratamento do câncer menos doloroso e difícil.

A equipe de enfermagem, por ser a principal responsável pela realização do CIP tem papel fundamental na prevenção de agravos relacionados à TIV. A avaliação criteriosa da criança é importante para que sejam reconhecidos os fatores predisponentes para a não obtenção do CIP na primeira tentativa.

A identificação desses fatores pode contribuir para a promoção de uma assistência mais qualificada em unidades oncológicas. Apropriados dessas informações, eles podem desenvolver estratégias e utilizar recursos para facilitar o CIP e aumentar a prevalência de 
obtenção na primeira tentativa. Isso poderá ser útil na redução de custos relacionados à repetição do procedimento, beneficiando a instituição de saúde.

\section{REFERÊNCIAS}

BEZERRA, A.R et al. "Minha punção venosa periférica": um material didático instrucional no preparo de crianças para o procedimento. Rev. Soc. Bras. Enferm. Ped. v.9, n.2, p.77-85 São Paulo, dezembro de 2009. Disponível em: http://www.sobep.org.br/revista/component/zine/article/120--minha-puno-venosa-perifricaum-material-didticoinstrucional-no-preparo-da-criana-para-o-procedimento.html>. Acesso em: 3 maio, 2016.

CARR, P.J et al. Development of a clinical prediction rule to improve peripheral intravenous cannulae first attempt success in the emergency department and reduce post insertion failure rates: the Vascular Access Decisions in the Emergency Room (VADER) study protocol. BMJ Open 2016;6:e009196. doi:10.1136/bmjopen-2015-009196. 2016b.

GOMES, A.V.O et al. A atuação do enfermeiro frente aos sentimentos e atitudes das crianças hospitalizadas submetidas à punção venosa periférica. Revenferm UFPE. v. 4 n. 1 p. 371-76, jan/mar 2010. Disponível em: < http://bases.bireme.br/cgi-

bin/wxislind.exe/iah/online/?IsisScript=iah/iah.xis\&src=google \&base=BDENF\&lang=p\&nex tAction=lnk\&exprSearch=18807\&indexSearch=ID> Acesso em: 25 abril, 2016.

LARSEN, $P$ et al. Pediatric peripheral intravenous access: does nursing experience and competence really make a difference? J Infus Nurs, v. 33, n. 4, p. 226-35, 2010.

LININGER, RA. Pediatric peripheral IV insertion success rates. PediatrNurs, v. 29, n. 5, p. 351-4, 2003.

LOON, F.H et al. Development of the A-DIVA Scale: A Clinical Predictive Scale to Identify Difficult Intravenous Access in Adult Patients Based on Clinical Observations. Medicine, v. 95, n. 16, 2016. Disponível em www.md-journal.com. Acesso em: 12 mai.

NEGRI, D.C, AVELAR, A.F.M., ANDREONI, S., PEDREIRA, M.L.G. Fatores predisponentes para insucesso da punção intravenosa periférica em crianças. Rev. LatinoAm. Enfermagem. v.20 n.6 [08 telas], 2012.

RIKER, M. W. et al. Validation and refinement of the Difficult Intravenous Access Score: a clinical prediction rule for identifying children with difficult intravenous access. Acad Emerg Med., v. 18, n. 11, p. 1129-1134, 2011.

SANTOS, L. M. Segurança do paciente pediátrico e sua família: estudo de tecnologias e eventos adversos relacionados à terapia intravascular periférica. Universidade Estadual de Feira de Santana (Projeto de pesquisa), 2014.

SZMUK, P. et al. The VeinViewer vascular imaging system worsens first-attempt cannulation rate for experienced nurses in infants and children with anticipated difficult intravenous access. AnesthAnalg., v. 116, n. 5, p. 1087-92. 2013.

VEZZANI, A et al. Ultrasonography as a guide during vascular access procedures and in the diagnosis of complications. J Ultrasound, v. 16, n. 4, p. 161-70, 2013.

YEN K; RIEGERT, A; GORELICK, M.H.Derivation of the DIVA Score A Clinical Prediction Rule for the Identification of Children With Difficult Intravenous Access Pediatric Emergency Care, v. 24, n. 3, 2008. 